Abbott, Lilly, Amgen, MSD, Will, Bio Minerals and Roche, Consultant for: Research support, consultant and/or speaker fees from: Pfizer, Abbott, Eli Lilly, Amgen, MSD, Roche, UCB, BMS, Novartis, Will-Pharma, Speakers bureau: Research support, consultant and/or speaker fees from: Pfizer, Abbott, Eli Lilly, Amgen, MSD, Roche, UCB, BMS, Novartis, Will-Pharma, Speakers bureau: Pfizer, Abbott, Lilly, Amgen, MSD, Will, Bio Minerals and Roche

DOI: 10.1136/annrheumdis-2019-eular.5349

\section{FRI0490 GOAL-DIRECTED TREATMENT OF OSTEOPOROSIS IN PATIENTS WITH RHEUMATOID ARTHRITIS USING DENOSUMAB FOR THREE YEARS}

Yuji Hirano $^{1}$, Yasuhide Kanayama ${ }^{2}$, Kyosuke Hattori $^{3}$, Daisuke Kihira ${ }^{1}$ Naoki Ishiguro ${ }^{3}$, Toshihisa Kojima ${ }^{3} .{ }^{1}$ Toyohashi Municipal Hospital, Rheumatology, Toyohashi, Japan; ${ }^{2}$ Toyota Kosei Hospital, Orthopaedic Surgery and Rheumatology, Toyota, Japan; ${ }^{3}$ Nagoya University School of Medicine, Orthopaedic Surgery and Rheumatology, Nagoya, Japan

Background: Osteoporosis (OP) is frequently concomitant with rheumatoid arthritis (RA). Effective treatment have to be performed in OP in RA (RAOP). Denosumab (DMB) is one of the promising drugs for the treatment of RAOP. We reported results of 12-month DMB treatment for RAOP from Japanese multicenter registry study (TBCR-BONE) in EULAR2016 1). Recently treatment goal of OP was reported from American society for bone and mineral research and National Osteoporosis Foundation (ASBMR-NOF) working group (Goal-Directed Treatment) ${ }^{2)}$. This progress report advocated that the goal of treatment is a T-score >2.5 at femoral neck, total hip (TH) or lumbar spine (LS) by DXA if the primary reason for starting treatment was T-score-2.5 at that skeletal site. The Working Group judged that it was intuitively reasonable to expect that initial treatment should offer at least a $50 \%$ chance of achieving the treatment goal within 3 to 5 years of starting therapy.

Objectives: The aim of this retrospective study is to evaluate whether 3year DMB treatment can achieve treatment goal of OP reported recently from TBCR-BONE.

Methods: This study used 78 female RAOP patients who completed 3year DMB treatment for RAOP. 36 patients in whom baseline T-score of LS-BMD were -2.5 or less were included in LS-BMD analysis. 52 patients in whom baseline T-score of TH-BMD were -2.5 or less were included in TH-BMD analysis in the same way. As was in clinical setting in Japan, $60 \mathrm{mg}$ DMB was administered every 6 months with vitamin D3 agent. Patients' characteristics, time course of T-score and baseline characteristics related to achievement of treatment goal (T-score>-2.5) were investigated.

Results: Baseline characteristics of 78 patients: Mean age was 71 years RA duration was 17 years. Prednisolone user was $32 \%$. LS-BMD analy sis $(n=35)$ T-score was significantly increased $(-3.5$ at baseline, -3.0 at 1 year, -2.9 at 2 years, -2.8 at 3 years). The rates of patients who achieved treatment goal were $30.6 \%$ at 1 year, $38.9 \%$ at 2 years and $44.4 \%$ at 3 years $($ Fig $1 \mathrm{~A})$. The achiever had significant lower baseline fracture risk by FRAX $(20.1 \%$ vs. $33.9 \%$ : $p=0.013)$, significant lower baseline $\mathrm{mHAQ}(0.45$ vs. 1.05: $\mathrm{p}=0.03)$ and significant lower baseline T-score of LS-BMD (-3.0 vs. -3.9 : $p<0.01)$ than the non-achiever did. TH-BMD analysis $(n=52)$ T-score was significantly increased $(-3.2$ at baseline, -3.0 at 1 year, -2.9 at 2 years, -2.9 at 3 years). The rates of patients who achieved treatment goal were $19.2 \%$ at 1 year, $28.8 \%$ at 2 years and $25.0 \%$ at 3 years (Fig1B). The achiever had significant lower baseline bone turn over marker (P1NP and TRACP-5b) and significant lower baseline T-score of TH-BMD (-2.6 vs. $-3.3: \mathrm{p}<0.01)$ than the non-achiever did. Cut-off values at baseline for achievement of treatment goal calculated using receiver operating characteristic analysis was -3.4 in LS-BMD and 2.7 in THBMD.

Conclusion: This study suggested that achievement of treatment goal in RAOP is not possible in LS-BMD and in TH-BMD when 3-year DMB was used as treatment for RAOP. Longer duration or earlier initiation of DMB treatment is necessary to achieve the goal. Although response in LS-BMD was related to fracture risk and physical function, response in TH-BMD was related to bone turnover. We will report 5-year results in the future.

\section{REFERENCES:}

[1] Hirano, et al. Ann Rheum Dis 2016; 75 (Suppl2): 94

[2] Cummings, et al. J Bone Miner Res 2017; 32: 3-10

Disclosure of Interests: Yuji Hirano: None declared, Yasuhide Kanayama: None declared, Kyosuke Hattori: None declared, Daisuke Kihira: None declared, Naoki Ishiguro Grant/research support from: AbbVie, Asahi Kasei, Astellas, Chugai, Daiichi-Sankyo, Eisai, Kaken, Mitsubishi Tanabe, Otsuka, Pfizer, Takeda, and Zimmer Biomet, Consultant for: Ono, Speak ers bureau: Astellas, Bristol-Myers Squibb, Daiichi-Sankyo, Eli Lilly, Pfizer, and Taisho Toyama, Toshihisa Kojima Grant/research support from: Chu gai Pharmaceutical (Investigator Initiated Study), Novartis, Nippon Kayaku, Eli Lilly, Eisai, Speakers bureau: Chugai Pharmaceutical, Takeda Pharmaceutical, Pfizer, Eli Lilly Japan, Bristol Myers Squibb, Ono Pharmaceutical, Daiichi Sankyo, Astelas, UCB, Janssen Pharmaceutical, Tanabe Mitsubishi,

DOI: 10.1136/annrheumdis-2019-eular.1889

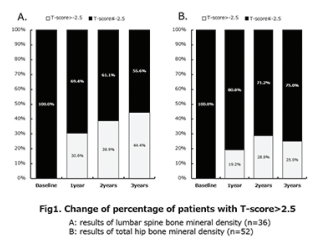

\section{FRI0491 MANAGEMENT OF SECONDARY OSTEOPOROSIS FRACTURES IN FRACTURE LIAISON SERVICE OF REIMS HOSPITAL IN FRANCE: STUDY AT 25 MONTHS OF FOLLOW-UP}

HITTINGER Ambre $^{1}$, Jean-Hugues Salmon ${ }^{1}$, Jean Paul Eschard ${ }^{2}$ Isabelle Charlot-Lambrecht ${ }^{1} .{ }^{1}$ Reims University Hospital, Rheumatology, Reims, France; ${ }^{1}$ Reims University Hospital, Rheumatology, Reims, France

Background: Osteoporosis is a major public health issue, progressing with the aging of the population and responsible for nearly 400,000 fractures/year in France. Fracture Liaison Services (FLS) are systems of detection and secondary prevention of osteoporotic fractures. These systems are recognized as effective by international scientific societies.

Objectives: The aim of this study was to evaluate the efficiency of Reims Hospital FLS protocol after 25 months of implementation.

Methods: We performed an ambispective monocentric observational study at the Reims Hospital between April 2016 and May 2018. We included patients over 50 years who were hospitalized in the orthopedic department for osteoporotic fractures. These patients were evaluated clinically, biologically and radiographically during a one-day hospitalization in the rheumatology unit. Therapeutic compliance and fracture recurrence were assessed in consultation at 3-month and 1-year telephone interviews.

Results: Sixty-four/242 patients $(26.4 \%)$ identified in orthopedic service were included. The average age was 72 years old with a sex ratio of $1: 10$. The most common fracture was the proximal end of the femur for 25 patients $(39 \%)$. Fifty-five patients $(85.9 \%)$ had an indication for osteoporosis treatment, the most prescribed was zoledronic acid for 45 patients $(81.9 \%)$. Twenty-four/55 patients $(43.6 \%)$ started their treatment and 22/24 $(91.7 \%)$ were still treated at one year.

One patient (1.63\%) died within the follow-up period. Twelve/55 patients (21.8\%) presented a new fall and $7 / 55$ patients $(12.7 \%)$ experienced a new fracture.

Comparative analysis of observant versus non-observant population groups at 1 year showed that non-observant patients had significantly more recurrent falls $(93.54 \%$ versus $68.18 \%, p=0.024)$ and received treatment of zoledronic acid (93.56\% versus $63.64 \%, p=0.010)$.

Further analysis showed that our low rate of initiation treatment was due to difficulties in implementing bisphosphonates, mainly because of the dental care needed. However, we observed a high rate of treatment adhesion at 1 year, once the treatment is started. During this study, we identified 2 areas of improvement: therapeutic education to improve patient adherence and fall risk factors to decrease recurrent fall rate.

Conclusion: There is a real benefit to the establishment of this FLS with a high rate of therapeutic compliance at 1 year follow-up. However there are opportunities for improvements in treatment initiation through patients therapeutic education.

\section{REFERENCES:}

[1] Lems WF, Dreinhöfer KE, Bischoff-Ferrari H, Blauth M, Czerwinski E, da Silva $J$, et al. EULAR/EFORT recommendations for management of patients older than 50 years with a fragility fracture and prevention of subsequent fractures. Ann Rheum Dis. mai 2017;76(5):802-10. 
Disclosure of Interests: Ambre HITTINGER: None declared, Jean-Hugues Salmon Speakers bureau: Janssen Novartis, jean paul eschard: None declared, Isabelle CHARLOT-LAMBRECHT: None declared DOI: 10.1136/annrheumdis-2019-eular.3033

\section{FRI0492 CALCULATING FRAX SCORE IN CLINICAL PRACTICE: PITFALLS AND PROBLEMS}

Navneet Kaur ${ }^{1}$, Barbara Mendez ${ }^{1}$, Avneet Vig ${ }^{2}$, Beverly Johnson ${ }^{1}$, Tony Francis ${ }^{3}$. ${ }^{1}$ Albert Einstein College of Medicine/Jacobi Medical Center, Rheumatology, Bronx, United States of America; ${ }^{2}$ Cleveland Clinic Martin Health, Rheumatology, Port St. Lucie, United States of America; ${ }^{3}$ Christiana Hospital, Nuclear Medicine, Newark, United States of America

Background: Osteoporosis related fractures cause significant morbidity and mortality. FRAX score uses clinical risk factors and country-specific data in addition to Bone Mineral Density (BMD) to assess patients with high 10-year risk of hip $(>3 \%)$ or major osteoporotic $(>20 \%)$ fracture. We noticed discrepancies between radiologist reported and physician calculated FRAX scores at our hospital. We hypothesized that providers are calculating FRAX score differently as BMD in the FRAX calculator is an optional input variable.

Objectives: This study was initiated to see the differences in results when FRAX score is calculated using T-score, BMD and no BMD and how this difference can influence treatment.

Methods: Retrospective chart review of 1200 DEXA reports from 2013 to 2015 was done. Patients between ages of 40-90 years with T-score ranging from $\leq-1$ to $>-2.5$ at femoral neck were included in the study. Patients already on osteoporosis therapy and/or with T-score $\leq-2.5$ or $>-$ 1 were excluded.

Pertinent risk factors were obtained from chart review. 237 patients met inclusion criteria and 3 separate FRAX scores were calculated using a) Femoral neck BMD b) T-score c) no BMD value. FRAX score reported by radiologist in the chart was also reviewed. Subsequently, FRAX scores obtained using T-score, no BMD value and radiology reported scores were compared against FRAX score calculated using femoral neck BMD (gold standard)

Results:

Table 1. shows demographic information for 237 patients who met inclusion criteria.

\begin{tabular}{lc}
\hline Demographics & \\
\hline Race & \\
Hispanic & $131(54.8 \%)$ \\
Black & $71(29.9 \%)$ \\
Caucasian & $15(6.3 \%)$ \\
Asian & $20(8.5 \%)$ \\
Average Age & $67 \pm 10.5$ years \\
Sex & \\
Male & $11(4.7 \%)$ \\
Female & $226(95.3 \%)$ \\
Average Height & $159.3 \pm 7.6 \mathrm{~cm}$ \\
Average Weight & $71 \pm 13 \mathrm{~kg}$ \\
BMl & $27.9 \pm 4.9$ \\
Previous fracture & $13(5.4 \%)$ \\
Current smoking & $14(5.9 \%)$ \\
Steroid use & $48(20.3 \%)$ \\
RA & $26(11 \%)$ \\
Secondary osteoporosis & $8(3.4 \%)$ \\
Alcohol use & $3(1.3 \%)$ \\
\hline
\end{tabular}

When FRAX score calculated using BMD was compared with FRAX score calculated without BMD, number of patients with high 10-year fracture probability decreased from 49 to 11 patients, which was a statistically significant decrease of $77.6 \%(p<0.001)$.

When data was stratified according to age, there was significant overestimation of risk in patients $>65$ years $(p<0.0001)$ when FRAX was calculated without BMD.

Conclusion: FRAX score calculation without BMD leads to both statistically and clinically significant overdiagnosis especially in elderly.

Interchanging $\mathrm{T}$ score and BMD to calculate FRAX score leads to same treatment decision.

Further education of providers regarding FRAX score is needed.

Many providers are not aware that if BMD column is left blank, it defaults the calculation to no BMD. A pop up alerting the user "no machine was selected so the calculation will default to no BMD which can lead to overestimation of risk" in FRAX tool might be helpful to avoid miscalculation.
REFERENCES:

[1] https://www.sheffield.ac.uk/FRAX/tool.jsp

[2] Prawiradilaga, RS, et al., "FRAX Calculated without BMD Resulting in a Higher Fracture Risk Than That Calculated with BMD in Women with Early Breast Cancer," Journal of Osteoporosis, vol. 2018, Article ID 4636028.

Table 2. shows difference in risk estimation when different FRAX score assessments were compared against gold standard femoral neck BMD.

\begin{tabular}{|c|c|c|c|c|}
\hline $\begin{array}{l}\text { FRAX Scores } \\
\text { Compared }\end{array}$ & $\begin{array}{c}\text { Discrepancy } \\
\text { in } \\
\text { Risk } \\
\text { estimation }\end{array}$ & Overestimation & Underestimation & $\begin{array}{c}\text { McNemar's } \\
\text { test }\end{array}$ \\
\hline $\begin{array}{l}\text { No BMD vs. BMD } \\
\text { FRAX }\end{array}$ & $52 / 237(16 \%)$ & 52 & 0 & $p<0.0001$ \\
\hline $\begin{array}{l}\text { Reported FRAX vs. } \\
\text { BMD } \\
\text { FRAX }\end{array}$ & $6 / 237(2.4 \%)$ & 2 & 4 & $\mathrm{p}=0.414$ \\
\hline $\begin{array}{l}\text { T-score vs. BMD } \\
\text { FRAX }\end{array}$ & $1 / 237(0.4 \%)$ & 1 & 0 & $\mathrm{p}=0.317$ \\
\hline
\end{tabular}

Disclosure of Interests: Navneet Kaur: None declared, Barbara Mendez: None declared, Avneet Vig: None declared, Beverly Johnson Shareholder of: ownership of johnson and johnson stock over 10,000 USD, Consultant for: I am a consultant for the rheumatology education group, Employee of: I have been paid indirectly by pharma as a consultant for the rheumatology education group, Tony Francis: None declared DOI: 10.1136/annrheumdis-2019-eular.1462

\section{FRI0493 CLINICLAL EFFICACY OF DENOSUMAB IN PATIENTS WITH OSTEOPOROSIS BETWEEN RHEUMATOID ARTHRITIS AND PRIMARY OSTEOPOROSIS; 24 MONTHS OF FOLLOW-UP}

Yasuhide Kanayama ${ }^{1}$, Taichi Tsuji ${ }^{2}$, Naohisa Futamura ${ }^{2}$, Naoaki Osada ${ }^{2}$, Hiroto Tachi ${ }^{2}$, Kazunori Todoroki ${ }^{2}$, Ryosuke Sugimoto2. ${ }^{1}$ Toyota Kosei Hospital, Orthopedic Surgery and Rheumatology, Toyota, Japan; ${ }^{2}$ Toyota Kosei Hospital, Orthopedic Surgery, Toyota, Japan

Background: Denosumab (DMB) is a fully human monoclonal antibody to the receptor activator of nuclear factor-kappaB ligand (RANKL) that blocks its binding RANK, inhibiting the development and activity of osteoclasts, decreasing bone resorption, increasing bone density and reducing fracture risk. Also $\mathrm{DMB}$ is a useful therapeutic agent for both rheumatoid arthritis (RA-OP) and primary osteoporosis (P-OP). However there is still few comparative study of clinical efficacy of DMB between RA-OP and P-OP. Objectives: To compare the clinical efficacy of $\mathrm{DMB}$ in patients with osteoporosis between rheumatoid arthritis and primary osteoporosis for 24 months.

Methods: RA patients diagnosed according to the 2010 ACR/EULAR crite ria. RA-OP and P-OP patients met at least one of the following criteria were eligible; a bone mineral density $\mathrm{T}$ score of $-2,5$ or less at the lumber spine or total hip and either one or more moderate or severe vertebral fractures or two or more mild vertebral fractures. All patients were initiated DMB from between October, 2013 and August, 2016. The final study cohort of 61 RA- OP and 50 P-OP patients received continuous DMB therapy more than 24 months. The DMB dose was $60 \mathrm{mg}$ at once every 6 months. In all cases native or activated vitamin $D$ has been used. We reviewed the results for 24 months about the increase and decrease of bone mineral density(BMD) of lumbar spine(LS) and total hip (TH) by DEXA and bone turnover markers, intact n-terminal propeptide type I procollagen(PINP) and tartrate-resistant acid phopshatate form $5 \mathrm{~b}$ (TRACP-5b).

Results: In the patients of RA-OP $(n=61)$ and P-OP $(n=50)$, the number of female was each $56(92 \%)$ and $45(90 \%)$ cases $(p=0.741)$. The mean age was $72.7 \pm 7.5$ and $76.8 \pm 7.8$ years old $(p=0.006)$; disease duration of RA-OP patients was $12.6 \pm 12.9$ years; the body mass index was $20.4 \pm 2.7$ and $20.3 \pm 3.0(p=0.687)$ and the FRAX was $27.2 \pm$ 15.7 and $24.6 \pm 11.1(\mathrm{p}=0.745)$. Clinical findings related to RA-OP at baseline were as follows; CRP $0.8 \pm 1.1$; DAS-CRP $3.28 \pm 1.36$; HAQ $1.16 \pm 1.04$ in RA-OP patients and in the patients of RA-OP and P-OP bone turnover markers and bone mineral density at baseline were as follows; P1NP $57.7 \pm 32.2$ and $64.0 \pm 32.7 \mu \mathrm{g} / \mathrm{l}(\mathrm{p}=0.151) ;$ TRACP-5b 563 